\title{
Proprioceptive Training to Improve Static and Dynamic Balance in Elderly
}

\author{
Giovanni Esposito ${ }^{1, *}$, Gaetano Altavilla ${ }^{1}$, Felice Di Domenico ${ }^{1}$, Sara Aliberti ${ }^{1}$, \\ Tiziana D'Isanto ${ }^{2}$ and Francesca D'Elia ${ }^{2}$ \\ ${ }^{1}$ Department of Political and Social Studies, University of Salerno, Italy \\ ${ }^{2}$ Department of Human, Philosophical and Education Sciences, University of Salerno, Italy
}

\begin{abstract}
Background: The risk of falls is a major cause of disability in older adults. A single fall, for the elderly, increases the risk of frequent falls and often causes an increased fear of falling again, which can become debilitating.

Objectives: The purpose of the present study was to test the effects of 12 weeks of proprioceptive training on the static and dynamic balance of older adults who have experienced at least one fall without compromising consequences.

Method: The sample consisted of older adults, aged 60 to 80 years, randomly divided into two groups: an experimental group, which followed a proprioceptive training protocol at a physiotherapy studio, and a control group, which did not observe any treatment. Static and dynamic balance assessment was performed pre-and post-intervention. The tests administered were the Berg Balance Test for the evaluation of static balance, and the Four-Square Step Test, for dynamic balance. A questionnaire to assess confidence and fear of falling was administered at the end of the protocol. Independent sample t-test was performed to analyze differences between groups and two-way ANOVA to test the null hypothesis of no change different over time between groups (interaction intervention $\times$ time). A Chi-Square was performed to analyze perceptions.

Conclusions: The results showed that 12 weeks of proprioceptive training effectively improved dynamic and static balance in older adults. The perceptions of the experimental group were more positive than the other one, in terms of the importance of physical activity to prevent the risk of falls, fear of falling again, and experience of falls during the last 12 weeks.
\end{abstract}

Keywords: Falls, Physical activity, Aging, Perceptions, Instability.

\section{INTRODUCTION}

Falls in the elderly are a public health priority because of their frequency and severity. A fall is an unintentional event that causes a person to move downward from the orthostatic position due to several causes [1]. They are the leading cause of home accidents and the leading cause of hospitalization and death. Each year, $1 / 3$ of people aged $>65$ years have at least one episode of a fall [2]. Therefore, falls occur predominantly in the elderly; the age group most affected is between 74 and 85 years. Most falls happen in the home (about $60 \%$ ), as both the elderly and disabled spend a lot of time there. Some comparisons have shown that, from the third age group onwards, i.e. from 65 years of age onwards, falls have increased by $30 \%$ in recent years. Not to mention that the elderly population, especially in Italy, has increased significantly. This is due to the increase in average life expectancy of men and women, respectively 80.8 and 85.2 years. However, with Covid-19, we see a slight reduction in average life expectancy of -1.3 years for men and -1 year for women, with a retreat of about ten years in life expectancy levels [3].

*Address correspondence to this author at the University of Salerno, via Giovanni Paolo II, 132- 84084, Fisciano (SA), Italy; Tel: 3490704840; E-mail: g.esposito198@studenti.unisa.it
The risk of falls is a major cause of disability in older adults [4]. Falls can be predictable, mainly due to people with walking problems or disoriented, accidental due to environmental factors, and unpredictable when the subject is in good clinical and functional condition. A single fall increases the risk of frequent falls for an older adult and often causes an increased fear of falling again, becoming debilitating. Another complication of the fall is then given by the post-fall anxiety syndrome, which leads the individual to reduce the movement, movements and daily activities, perhaps carried out in an overly cautious way, just for fear of falling. This factor contributes to reduced self-esteem and muscle strength, promoting abnormal walking and, in the long run, further increasing the risk of falling and social isolation [5]. According to World Health Organization [6] guidelines, the recommended physical activity levels from the third age onwards are at least 150 minutes of moderate to vigorous aerobic physical activity per week. Sessions must predict at least ten minutes of aerobic activity; joint mobility exercises to improve balance and prevent the risk of falls three or more times a week; muscle strengthening, at least twice a week. Given the disabling impact of these accidental events, it is important to maintain constantly active assessment and preventive management programs of fall risk. 
Modifiable risk factors include poor balance, which can be improved through physical activity. Falling is related to losing balance, so exercises to improve balance play an important role in fall prevention and correct posture [7]. Individuals who report a fall or are considered at risk for falling should first undergo a balanced assessment. Some of the well-validated tests to predict falls are the Berg Balance Scale [8], to assess static balance and The Four square step test (FSST), to assess dynamic balance in the elderly [9]. As the problem of balance is associated with proprioceptive decline, one of the most useful types of training is the proprioceptive one, as awareness of one's body position is fundamental for communication with the environment. Proprioception allows for body stability and orientation during static and dynamic activities. Several studies have proposed different training protocols that significantly improve balance ability [10,11]. However, further studies on the content and effectiveness of various proprioceptive exercise programs are needed [12]. The purpose of the present study was to test the effects of 12 weeks of proprioceptive training on the static and dynamic balance of older adults who experienced at least one fall without compromising consequences and to analyze their perceptions toward the activity performed and fear of falling.

\section{MATERIAL AND METHODS}

\section{Design and Participants}

The sample consisted of 30 Italian elderly (age, mean $\pm \mathrm{SD}=71 \pm 4,9$ years old), randomly divided into two groups: an experimental group and a control group, composed of 15 elderly. $60 \%$ were women. Inclusion criteria were to have experienced at least one nondebilitating fall, to be able to perform the tests proposed in this study and to be mostly sedentary. Exclusion criteria were older adults with cognitive impairment or health problems, only if the condition could impair their ability to cooperate or was associated with rapid deterioration. Informed consent was obtained from all participants. Data were treated anonymously.

\section{Test Procedure}

\section{Berg Balance Test}

The Berg Balance Test (BBT) was performed pre and post 12 weeks to assess static balance. The BBS consisted of 14 items scored on a 5-point ordinal scale, ranging from 0 to 4 ( 0 indicating the lowest level of function; 4 indicating the highest level of function), with a maximum total score of 56 . A score of 56 indicated functional balance. A score of $<45$ indicated individuals might be at greater risk of falling. Equipment required for testing were a stopwatch and a ruler or other 2, 5, and 10 inches indicators.

\section{Item Description Score (0-4)}

1. Sitting to standing

2. Standing unsupported

3. Sitting unsupported

4. Standing to sit

5. Transfers

6. Standing with eyes closed

7. Standing with feet together

8. Reaching forward with an outstretched arm

9. Retrieving object from the floor

10. Turning to look behind

11. Turning 360 degrees

12. Placing an alternate foot on a stool

13. Standing with one foot in front

14. Standing on one foot

\section{Four Square Step Test}

Four Square Step Test (SSST) was performed pre and post 12 weeks to assess dynamic balance. The subject was required to step sequentially over four rods arranged in a cross on the ground. At the beginning of the test, the subject was in square 1, facing square 2 . The objective was to enter each square as quickly as possible with both feet in the following sequence: square $2,3,4,1,4,3,2,1$. Two tests were performed, and the best time (in seconds) was taken as the score. The timing starts when the first foot touches the floor in square 2 and ends when the last foot touches the floor again in square 1 . If the score was $>15$ seconds, this meant there was an increased risk of falling.

\section{Questionnaire}

A questionnaire to assess confidence and fear of falling was administered at the end of 12 weeks. The questionnaire was administered in paper form and included four items regarding physical activity and fall risk. Each item could be answered Yes/No. 
1. Do you consider the practice of physical activity important?

2. Do you think physical activity helps prevent the risk of falls?

3. Are you afraid of falling?

4. Have you experienced other falls during the last 12 weeks?

\section{Training Protocol}

\section{Experimental Group}

The experimental group followed a proprioceptive training protocol for 12-week intending to improve static and dynamic balance. The elderly came to a physiotherapy studio twice a week, and the sessions lasted 60 minutes. The proposed exercises were adapted for each older adult and included proprioceptive exercises requiring instability management and postural exercises. The methodology used in the central phase was the functional circuit, repeated three times. A detailed description of the protocol is shown in Table 1.

\section{Statistical Analysis}

After verifying the normality of the data with the Shapiro-Wilk test and homogeneity of variances with the Levene test, an independent sample t-test was performed to analyze differences between groups. A two-way repeated-measures analysis of variance
(ANOVA) was performed to test for differences in proprioceptive training-induced changes in static and dynamic balance in the elderly. The independent variables included one between-subjects factor (training intervention) with two levels (experimental and control group) and one within-subject factor (time) with two levels (pre-and post-12 weeks-intervention). To examine the influence of training intervention on the development of our dependent variables, we used these ANOVAs to test the null hypothesis of no different change over time between groups (training intervention $x$ time interaction). To qualitatively interpret the magnitude of differences, effect sizes (d) and associated $95 \%$ confidence intervals $(95 \% \mathrm{Cl})$ were classified as small $(0.2-0.5)$, moderate $(0.5-0.8)$ and large $(>0.8)$. A Chi-Square analysis $\left(X^{2}\right)$ was performed to analyze differences in perceptions between two groups post-intervention. Significance was set at $P<$ 0.05. Data were analyzed using SPSS (IBM SPSS Statistics for Windows, Version 25.0. Armonk, NY).

\section{RESULTS}

The mean and standard deviation (SD) results of the BBT and FSST test measurements administered on entry and exit in the experimental (EXP) and control (CON) group are shown in Table 2.

From the repeated-measures ANOVA analysis, we can appreciate for the experimental group, the existence of a statistically significant difference in both BBT and FSST, respectively: $(\mathrm{d}[\mathrm{Cl} 95 \%]=0.92[0.80$; 0.96 ] and 0.87 [0.67; 0.92]; $P<0.05)$. In contrast, no

Table 1: Proprioceptive Training Protocol

\begin{tabular}{|c|c|c|c|c|}
\hline & Exercise & Set & Reps & Rest \\
\hline \multirow[t]{4}{*}{ Warm-up } & $\begin{array}{l}\text { 1. Standing with hands resting on the side, open and close the side } \\
\text { with the breaths }\end{array}$ & 1 & 2 minutes & $1^{\prime}$ \\
\hline & $\begin{array}{l}\text { 2. Standing with hands on the iliac crests, mobilization of the pelvis } \\
\text { in anterior and retroversion with breaths }\end{array}$ & 1 & 2 minutes & $1^{\prime}$ \\
\hline & 3. Prone decubitus: cat stretch & 1 & 2 minutes & $1^{\prime}$ \\
\hline & 4. Bird dog & 1 & 2 minutes & $2^{\prime}$ \\
\hline \multirow[t]{6}{*}{ Central phase } & Circuit & & & \\
\hline & \multirow{5}{*}{$\begin{array}{l}\text { - Balance on one leg (stork position) } \\
\text { - Get up from a } 60 \mathrm{~cm} \text { highchair } \\
\text { - Walking on a proprioceptive pad } \\
\text { - Walk-in a straight line of } 10 \text { meters } \\
\text { - Throw softballs towards a wall } 10 \text { feet away }\end{array}$} & \multirow{5}{*}{3} & 10" per leg & \multirow{5}{*}{$1^{\prime}$} \\
\hline & & & 5 & \\
\hline & & & 10 per leg & \\
\hline & & & 1 & \\
\hline & & & 10 & \\
\hline \multirow[t]{3}{*}{ Cool down } & 1. Walking & 1 & 5 minutes & $1^{\prime}$ \\
\hline & $\begin{array}{l}\text { 2. Supine decubitus (breathing with the perception of every part of } \\
\text { the body) }\end{array}$ & 1 & 5 minutes & $1^{\prime}$ \\
\hline & 3. Stretching & 1 & 5 minutes & $1^{\prime}$ \\
\hline
\end{tabular}


Table 2: BBT and FSST Score before and after 12 Weeks

\begin{tabular}{|c|c|c|c|c|c|c|c|c|}
\hline \multirow{3}{*}{ Samples } & \multicolumn{4}{|c|}{$\operatorname{EXP}(n=15)$} & \multicolumn{4}{|c|}{$\operatorname{CON}(n=15)$} \\
\hline & \multicolumn{2}{|c|}{ Pre (sec) } & \multicolumn{2}{|c|}{ Post (sec) } & \multicolumn{2}{|c|}{ Pre (sec) } & \multicolumn{2}{|c|}{ Post (sec) } \\
\hline & BBT & FSST & BBT & FSST & BBT & FSST & BBT & FSST \\
\hline 1 & 45 & 50 & 14 & 13 & 38 & 35 & 26 & 28 \\
\hline 2 & 48 & 55 & 15 & 10 & 31 & 30 & 20 & 21 \\
\hline 3 & 30 & 35 & 25 & 21 & 46 & 47 & 18 & 20 \\
\hline 4 & 41 & 48 & 13 & 9 & 55 & 55 & 20 & 19 \\
\hline 5 & 38 & 45 & 25 & 18 & 32 & 30 & 25 & 25 \\
\hline 6 & 35 & 42 & 15 & 10 & 58 & 55 & 15 & 15 \\
\hline 7 & 39 & 46 & 26 & 17 & 40 & 42 & 18 & 17 \\
\hline 8 & 45 & 50 & 18 & 13 & 32 & 33 & 28 & 18 \\
\hline 9 & 50 & 57 & 15 & 9 & 35 & 39 & 20 & 22 \\
\hline 10 & 32 & 44 & 21 & 17 & 30 & 28 & 27 & 28 \\
\hline 11 & 35 & 40 & 25 & 20 & 51 & 51 & 15 & 16 \\
\hline 12 & 29 & 35 & 29 & 24 & 31 & 30 & 22 & 21 \\
\hline 13 & 33 & 38 & 21 & 16 & 32 & 33 & 19 & 20 \\
\hline 14 & 51 & 56 & 16 & 14 & 53 & 54 & 14 & 15 \\
\hline 15 & 45 & 54 & 29 & 23 & 47 & 46 & 15 & 14 \\
\hline $\begin{array}{c}\text { Mean } \\
\text { (SD) }\end{array}$ & $\begin{array}{l}39.7 \\
(7.3)\end{array}$ & $\begin{array}{l}46.3 \\
(7.3)\end{array}$ & $\begin{array}{l}20.4 \\
(5.6)\end{array}$ & $\begin{array}{l}15.6 \\
(4.9)\end{array}$ & $\begin{array}{l}40.7 \\
(9.9)\end{array}$ & $\begin{array}{c}40.5 \\
(10.0)\end{array}$ & $\begin{array}{l}20.1 \\
(4.6)\end{array}$ & $\begin{array}{l}19.9 \\
(4.4)\end{array}$ \\
\hline
\end{tabular}

statistically significant difference was appreciated in the control group. A significant group $\mathrm{x}$ moment interaction can be appreciated in both BBT variable and FSST variable, respectively: $(\mathrm{d}[\mathrm{Cl} 95 \%]=0.76[0.58 ; 0.84]$ and 0.49 [0.20; 0.65]; $P<0.05)$. A detailed description is shown in Table 3.

Two significant associations were found between two groups (EXP and CON) and fear of falling $\left(X^{2}=\right.$ $7,03 ; p=0.01)$, and experience of falling $\left(X^{2}=7,50\right.$; $p=0.00$ ), in the last 12 weeks. A detailed description is shown in Table 4.

\section{DISCUSSION}

The results showed that twelve weeks of proprioceptive training improved both static and dynamic balance in the elderly group. From the repeated-measures ANOVA analysis, we can appreciate for the experimental group, the existence of a statistically significant difference in both the BBT and FSST, respectively: $(\mathrm{d}[\mathrm{Cl} 95 \%]=0.92[0.80 ; 0.96]$ and 0.87 [0.67;0.92]; $P<0.05$ ). In contrast, no statistically significant difference was appreciated in the control group. Having a good balance means having a good foundation that keeps the body erect and stable [13]. The proposed proprioceptive circuit protocol improved both static and dynamic balance in twelve weeks. Another study [14], which compared utilized the functional circuit methodology, also proved effective in improving balance in the elderly. According to Sherrington et al., [15, 16], exercises performed for fall prevention should focus on balance to strengthen abdominal muscles and posture. From the Chi-square analysis, we can see that there are no differences in perception regarding the importance of physical activity and the usefulness of physical activity in preventing the

Table 3: Repeated-Measures ANOVA between EXP and CON Group on Entry and Exit

\begin{tabular}{|c|c|c|c|c|c|c|}
\hline \multirow[b]{2}{*}{ Test } & \multicolumn{2}{|c|}{$\operatorname{EXP}(n=15)$} & \multicolumn{2}{|c|}{$\operatorname{CON}(n=15)$} & \multicolumn{2}{|c|}{ Moment $\times$ Group Interaction } \\
\hline & $p$ & d (95\% Cls) & $p$ & d (95\% Cls) & $p$ & d (95\% Cls) \\
\hline BBT & 0.001 & $0.92(0.80 ; 0.95)$ & 0.695 & $0.01(-0.02 ; 0.24)$ & 0.001 & $0.76(0.58 ; 0.84)$ \\
\hline FSST & 0.001 & $0.87(0.67 ; 0.92)$ & 0.796 & $0.00(-0.100 .21)$ & 0.001 & $0.49(0.20 ; 0.65)$ \\
\hline
\end{tabular}


Table 4: Differences in Perceptions between EXP and CON Group Post 12 Weeks

\begin{tabular}{|c|c|c|c|c|c|}
\hline \multirow{2}{*}{ Variable } & \multirow{2}{*}{ Options } & \multicolumn{2}{|c|}{ Groups } & \multicolumn{2}{|c|}{ Chi-squared analysis } \\
\hline & & EXP & CON & $x^{2}$ & $P$ \\
\hline \multirow{2}{*}{ 1. Do you consider the practice of physical activity important? } & Yes & 13 & 10 & 1.67 & 0.19 \\
\hline & No & 2 & 5 & & \\
\hline \multirow{2}{*}{ 2. Do you think physical activity helps prevent the risk of falls? } & Yes & 13 & 9 & 2.72 & 0.10 \\
\hline & No & 2 & 6 & & \\
\hline \multirow{2}{*}{ 3. Are you afraid of falling? } & Yes & 6 & 13 & 7.03 & 0.01 \\
\hline & No & 9 & 2 & & \\
\hline \multirow{2}{*}{ 4. Have you experienced other falls during the last 12 weeks? } & Yes & 0 & 5 & 7.50 & 0.00 \\
\hline & No & 16 & 10 & & \\
\hline
\end{tabular}

risk of falls between the experimental group and the control group.

Interestingly, things have changed today, as in previous years, older adults tended to cut back on physical activity due to fear of injury. This could happen because today's elderly have gained more confidence and security as training is adapted and customized to their needs [17]. There is, however, a difference in fear of falling again. The experimental group, who followed a proprioceptive training protocol to improve static and dynamic balance, reported that they had no fear of falling again. Probably proprioceptive training developed greater confidence in the elderly and awareness of their bodies. Not surprisingly, comparing the balance results in and out of a test, we see important improvements. Most of the control group, on the other hand, stated that they were afraid of falling, as they did not engage in any physical activity during twelve weeks. Therefore, they did nothing to try to improve their condition; not surprisingly, the results of the entry and exit balance tests did not change much. Another difference in perception between the two groups concerns the actual falls experienced during the last twelve weeks. In contrast to the control group, where there were some falls by some subjects, no older adults experienced a fall in the experimental group.

According to reviews $[16,18]$, older adults who regularly engage in physical activity are less likely to fall than inactive peers, especially exercises that focus on balance and functional training to improve stability and coordination. The study has some limitations, including sample size, emphasizing the need to repeat the study in a larger sample. Adequate proprioceptive training is important for maintaining physical fitness, preventing the risk of falls, and improving static and dynamic balance in the elderly [19]. This protocol can be used as a preventive measure, in addition to other training sessions since, according to World Health Organization guidelines [6], for the third age, sessions should include strength, endurance, mobility, and balance.

\section{CONCLUSION}

Proprioceptive training, administered twice a week for twelve weeks, significantly improved static and dynamic balance in the over-65 elderly group. The perceptions of the experimental group were also more positive than the control group, in terms of the importance of physical activity to prevent the risk of falls, fear of falling again, and experience of falls during the last 12 weeks, the period during which the proprioceptive protocol was administered. In summary, the elderly seem to have gained more confidence from proprioceptive training. Future studies could expand the sample and reproduce the protocol with the questionnaire to have more representative results.

\section{CONFLICTS OF INTEREST}

There are no conflicts of interest.

\section{REFERENCES}

[1] The prevention of falls in later life. A report of the Kellogg International Work Group on the Prevention of Falls by the Elderly. Danish medical bulletin 1987; 34 Suppl 4: 1-24.

[2] Istituto Superiore di Sanità. Le cadute negli anziani. Epicentro ISS. Consulted on 22 November 2021. https://www.epicentro.iss.it/incidenti-domestici/rischi-caduteanziani

[3] Istat. Le Condizioni di Salute Della Popolazione Anziana in Italia. 2019. Consulted on 22 November 2021. Available at https://www.istat.it/it/files//2021/07/Report-anziani-2019.pdf

[4] Karlsson MK, Magnusson H, von Schewelov T, Rosengren BE. Prevention of falls in the elderly--a review. Osteoporosis international: a journal established as result of cooperation 
between the European Foundation for Osteoporosis and the National Osteoporosis Foundation of the USA 2013; 24(3): 747-762. https://doi.org/10.1007/s00198-012-2256-7

[5] Boushon B, Nielsen G, Quigley P, Rutherford P, Taylor J, Shannon D, Rita S. How-to Guide: Reducing Patient, Injuries from Falls, Cambridge, in MA: Institute for Healthcare Improvement 2012. Available at www.ihi.org

[6] WHO. Physical Activity Strategy for the WHO European Region 2016-2025; WHO Regional Office for Europe: Vilnus, Lithuania 2015. Consulted in 22 November 2021. https://www.euro.who.int/_data/assets/pdf_file/0010/282961 /65wd09e_PhysicalActivityStrategy_150474.pdf

[7] Aliberti S, Invernizzi PL, Scurati R, D'Isanto T. Posture and skeletal muscle disorders of the neck due to the use of smartphones. Journal of Human Sport and Exercise 2020; 15(3proc): S586-S598. https://doi.org/10.14198/jhse.2020.15.Proc3.11

[8] Berg K, Wood-Dauphine SL, Williams JL, Gayton D. Measuring balance in the elderly: validation of an instrument Canadian Journal of Public Health 1992; S2: s7-s11.

[9] Dite W, Temple VA. A clinical test of stepping and change of direction to identify multiple falling older adults. Archives of physical medicine and rehabilitation 2002; 83(11): 15661571. https://doi.org/10.1053/apmr.2002.35469

[10] Martínez-Amat A, Hita-Contreras F, Lomas-Vega R, Caballero-Martínez I, Alvarez PJ, Martínez-López E. Effects of 12-week proprioception training program on postural stability, gait, and balance in older adults: a controlled clinical trial. Journal of strength and conditioning research 2013; 27(8): $2180-2188$ https://doi.org/10.1519/JSC.0b013e31827da35f

[11] Espejo-Antúnez L, Pérez-Mármol JM, Cardero-Durán $M$, Toledo-Marhuenda JV, Albornoz-Cabello M. The Effect of Proprioceptive Exercises on Balance and Physical Function in Institutionalized Older Adults: A Randomized Controlled Trial. Archives of physical medicine and rehabilitation 2020; 101(10): 1780-1788.

https://doi.org/10.1016/j.apmr.2020.06.010
[12] Ferlinc A, Fabiani E, Velnar T, Gradisnik L. The Importance and Role of Proprioception in the Elderly: a Short Review. Materia socio-medica 2019; 31(3): 219-221. https://doi.org/10.5455/msm.2019.31.219-221

[13] Picone $A D$, lona $T$, Rigon M, Aliberti S. The importance of balance with the prescriptive teaching in kickboxing. Journal of Human Sport and Exercise 2021; 16(4proc): S2023S2030.

https://doi.org/10.14198/jhse.2021.16.Proc4.49

[14] Cilento MDBR. Avaliação da eficácia de protocolos de treinamento da atividade sentado-para-de-pé em mulheres idosas. Fisioterapia Brasil 2005; 6(6): 412-418. https://doi.org/10.33233/fb.v6i6.2032

[15] Sherrington C, Tiedemann A, Fairhall N, Close JC, Lord SR. Exercise to prevent falls in older adults: an updated metaanalysis and best practice recommendations. New South Wales public health bulletin 2011; 22(3-4): 78-83. https://doi.org/10.1071/NB10056

[16] Sherrington C, Fairhall NJ, Wallbank GK, Tiedemann A Michaleff ZA, Howard K, Clemson L, Hopewell S, Lamb SE. Exercise for preventing falls in older people living in the community. The Cochrane database of systematic reviews 2019; 54(15): 885-891. https://doi.org/10.1002/14651858.CD012424.pub2

[17] Lanzara I, Aliberti S, Cereda F. Self-perception on physical activity of elderly people. Journal of Human Sport and Exercise 2021; 16(4proc): S1705-S1712. https://doi.org/10.14198/jhse.2021.16.Proc4.17

[18] Aliberti S, Raiola G. Effects of Line Dancing on Mental Health in Seniors after COVID-19 Pandemic. Educ. Sci. 2021; 11: 677. https://doi.org/10.3390/educsci11110677

[19] Sannicandro I, Cofano G, Rosa AR, Raiola G. Sedentary conditions during the lockdown and movement opportunities for the Italian elderly. MOJ Sports Med. 2020; 4(3):87-90. http://hdl.handle.net/11369/388156

https://doi.org/10.6000/1929-6029.2021.10.18

(c) 2021 Esposito et al.; Licensee Lifescience Global.

This is an open access article licensed under the terms of the Creative Commons Attribution License (http://creativecommons.org/licenses/by/4.0/) which permits unrestricted use, distribution and reproduction in any medium, provided the work is properly cited. 